\title{
Consumo de tabaco en médicos residentes de pediatría en la Argentina. Prevalencia actual y tendencia en los últimos diez años
}

\author{
Tobacco consumption among pediatric residents in Argentina. \\ Current prevalence and trend over the past 10 years
}

\author{
Dr. Emiliano Gigliottia ${ }^{a}$ Dr. Fernando Ferrero ${ }^{a}$, Dr. Claudio Castaños ${ }^{b}$, \\ Dra. María Teresa Blenginic, Dr. Pablo Durán ${ }^{a}$, Dra. Laura Moreno ${ }^{c, d}$ y \\ Grupo de estudio del tabaquismo en la residencia de pediatría*
}

\section{RESUMEN}

Introducción. Los pediatras se encuentran en una posición estratégica para actuar en la prevención del tabaquismo.

Objetivos. Estimarla prevalencia del tabaquismo en residentes de pediatría, analizar los factores asociados, describir su conducta preventiva y evaluar las diferencias en la última década.

Métodos. Estudio transversal, por encuesta anónima, autoadministrada, que incluyó a residentes de pediatría de ocho hospitales de la Argentina. Se evaluaron el hábito de fumar, los factores asociados, la actitud hacia el consumo de tabaco de los pacientes o los padres, y se compararon los resultados con los de 2002.

Resultados. De 448 encuestados, 20,1\% fumaban. Nohubodiferenciassignificativasentrefumadores y no fumadores respecto del sexo, los hijos, el número de guardias y el tener un jefe fumador. El padre fumador fue un factor de riesgo para tabaquismo solo en las mujeres (OR 1,98; IC 95\% $1,09$ a 3,$61 ; p=0,01)$. Los que vivían en pareja fumaban menos (OR 0,57; IC 95\% 0,34 a 0,96; $p=0,03$ ). Solo $18,1 \%$ refirieron tener una conducta activa frente al tabaquismo de los pacientes, sin diferencias entre fumadores y no fumadores. No hubo diferencia con la prevalencia del tabaquismo de 2002, pero sí mayor proporción de residentes que aconsejaban (32,4\% contra $26,1 \% ; p<0,01)$ y advertían a sus pacientes $(37,7 \%$ contra $18,6 \% ; p<0,01)$, y que recibieron información durante su formación $(63,6 \%$ contra $39,8 \% ; p<0,01)$.

Conclusiones. El 20,1\% de los médicos residentes de pediatría eran fumadores, siendo más frecuente en las mujeres con un padre fumador. La conducta activa frente al tabaquismo de los pacientes o sus padres fue muy baja. Pese a las políticas oficiales, el tabaquismo en este grupo no se ha modificado en la última década, aunque aumentó la proporción de quienes recibieron información durante su formación, y aconsejan $\mathrm{y}$ advierten a los pacientes sobre sus riesgos. Palabras clave: internado y residencia, tabaco, tabaquismo, cese del tabaquismo, rol profesional.

http:/ /dx.doi.org/10.5546/aap.2013.315

\section{INTRODUCCIÓN}

El tabaco constituye la principal causa de muerte prevenible; se estima que ocasiona más de 5 millones de muertes anuales en el mundo. ${ }^{1}$ Debido a la morbimortalidad que genera, el tabaquismo implica un costo económico significativo, que puede explicar hasta el 15\% del gasto en salud. ${ }^{2}$

Los médicos se encuentran en una posición estratégica para influenciar sobre el hábito de fumar en sus pacientes. ${ }^{3}$ Esto es particularmente trascendente entre los pediatras, ya que pueden actuar en la prevención del inicio del consumo de tabaco en los niños y adolescentes, y sobre sus padres para incentivar el abandono del hábito, ${ }^{4}$ lo que reduce la exposición de los niños al humo de tabaco ambiental. ${ }^{5}$ Ludovica. La Plata, Buenos Aires.

i. Hospital Gutiérrez. C.A.B.A.

Correspondencia:

Dr. Emiliano Gigliotti: emigigliotti@hotmail. com

Conflicto de intereses: Ninguno que declarar.

Recibido: 9-10-2012

Aceptado: 16-2-2013

\section{*Grupo de estudio del tabaquismo en la residencia de pediatría:}

Dra. Lina Abrame, Dra. María Carolina Amvegc 'Dra. Paola Biancottic, Dra. María T. Blenginic, Dr. Claudio Castaños ${ }^{b}$, Dra. Laura Castro ${ }^{a}$, Dra. Silvina Ciprianif, Dr. Pablo Collettie , Dr. Pablo Durán ${ }^{a}$, Dr. Gastón Fernández $z^{g}$, Dr. Fernando Ferrero ${ }^{a}$, Dra. Patricia Fignanac, Dra. Ana Figueroaf, Dr. Emiliano Gigliotti ${ }^{a}$, Dra. Laura Moreno $^{c, d}$, Dra. Mercedes Posadas ${ }^{b}$, Dra. Andrea Reinosog, Dr. Fernando Rentería ${ }^{h}$, Dra. Viviana Rodríguezi, Dra. Bárbara Sparrow ${ }^{i}$ Dra. Erica Stelmaszewskib y Dr. Jorge Urrutigoytis 
Debido a la particular carga de trabajo de la residencia y al estrés a que se encuentran sometidos, estos profesionales podrían verse más expuestos al consumo de tabaco. ${ }^{6}$ En el año 2002 evaluamos el hábito de fumar en residentes de pediatría de la Argentina y encontramos que $22,1 \%$ de ellos fumaban. ${ }^{7}$ En concordancia con los lineamientos del Convenio Marco de la Organización Mundial de la Salud (OMS) para el Control del Tabaco (CMCT) ${ }^{8}$ en la Argentina se han implementado distintas políticas relacionadas con el tema y se logró, en los últimos 10 años, un descenso en la prevalencia de consumo de tabaco de $39,8 \%{ }^{9}$ a $27,1 \%{ }^{10}$ Sería de esperar que después de las acciones gubernamentales la prevalencia del tabaquismo entre los médicos residentes disminuyera al menos en la misma proporción.

El objetivo del presente trabajo fue estimar la prevalencia del hábito de fumar en médicos residentes de pediatría, evaluar los posibles factores de riesgo asociados, describir la conducta de los profesionales encuestados en relación con el tabaquismo de los pacientes o sus padres, y comparar los resultados con los obtenidos en 2002.

\section{MATERIAL Y MÉTODOS}

Estudio transversal, por encuesta anónima, autoadministrada y cerrada.

Se llevó a cabo durante el mes de mayo de 2011 en los ocho hospitales pediátricos de la Argentina que participaron en un estudio anterior: ${ }^{7}$ Elizalde, Garrahan y Gutiérrez (Ciudad Autónoma de Buenos Aires), Santísima Trinidad e Infantil (Córdoba), Castro Rendón (Neuquén), Notti (Mendoza) y Sor María Ludovica (La Plata). Se incluyeron todos los médicos residentes de pediatría presentes en su hospital el día de la encuesta que aceptaran participar en la investigación y se excluyeron aquellos que no se encontraban prestando servicios en la institución en el momento de la encuesta (licencia ordinaria, enfermedad, rotación, trámites, etc.).

Las variables de estudio fueron sexo; edad; hábito de fumar; año de la residencia en curso; número de guardias semanales; convivientes, si la madre, el padre o su jefe inmediato fumaban; edad de comienzo; lugar y actividades del hospital en las que más fumaba; si había aumentado el consumo de tabaco desde el ingreso en la residencia; si había recibido información durante su residencia o formación universitaria sobre cómo intervenir en el consumo de tabaco de los pacientes, y su actitud hacia el consumo de tabaco de sus pacientes o sus padres (si preguntaba, advertía sobre los riesgos y aconsejaba la cesación o el no inicio del tabaquismo). Para el análisis se construyó la variable dicotómica "conducta activa" asignándole un valor afirmativo solo si el sujeto respondía "siempre" a las tres preguntas sobre conducta (preguntar, advertir y aconsejar).

A los efectos del presente trabajo se definió como fumador a aquel que a lo largo de su vida hubiera fumado al menos 100 cigarrillos, 20 cigarros o 20 pipas, y que fumaba en el momento de la encuesta.

Tamaño muestral: considerando que existen alrededor de 1200 médicos residentes de pediatría en el país, que la prevalencia de tabaquismo en ellos se sitúa en $22 \% \pm 5 \%$ y que hasta $10 \%$ podrían negarse a participar, se estimó un tamaño muestral de 230 sujetos para alcanzar un nivel de confianza del $95 \%$. Se trató de una muestra de conveniencia, debido a que participaron sujetos pertenecientes a los mismos programas de residencia que lo hicieron en el año 2002 a fin de poder comparar los resultados. La muestra seleccionada abarca aproximadamente el $50 \%$ de los residentes de pediatría del país. Se estimó que al menos dos tercios de ellos se encontrarían en condiciones de participar.

Análisis estadístico: la distribución de las respuestas a cada variable se efectuó por medio de porcentajes con sus intervalos de confianza del 95\% o media y desviación estándar, según correspondiera. Se valoró la asociación entre cada uno de los predictores y la variable de resultado por medio de la prueba de la $\chi^{2}$ o la prueba exacta de Fisher (según correspondiera), calculando los OR con sus respectivos intervalos de confianza del $95 \%$. Para las variables numéricas se valoró la diferencia entre medias mediante la prueba de la $\mathrm{T}$, si presentaban distribución normal, y la prueba de la U de Mann-Whitney, en caso contrario. Finalmente, se construyeron dos modelos de regresión logística. Uno valoró el consumo de tabaco en los residentes incluido el resto de las variables; el otro modelo exploró la conducta de los médicos residentes hacia el tabaquismo de sus pacientes incluidos edad, sexo, tabaquismo personal y en sus padres, e información recibida.

La diferencia entre los resultados del presente estudio y los alcanzados en el estudio de 2002 se evaluó por medio de las pruebas de la T o de la $\chi^{2}$, según correspondiera. Se consideró un nivel de significación de 0,05 de dos colas. Los datos se analizaron con SPSS 17.0 (SPSS Inc., Chicago, EE.UU., 2002).

Consideraciones éticas: se solicitó y obtuvo la aprobación de los Comités de Ética o 
Investigación de cada institución, de acuerdo con las normativas propias de cada ámbito. Al finalizar la encuesta, se entregó a los participantes material sobre los riesgos del hábito de fumar, contacto para asesoramiento sobre cesación de tabaquismo y una guía sobre la conducta del profesional frente al tabaquismo de los pacientes. Todos los datos fueron recabados de forma anónima y se garantizó su confidencialidad.

\section{RESULTADOS}

Durante mayo de 2011 se llevó a cabo la encuesta, encontrándose presentes en sus hospitales $480(69,7 \%)$ de los 689 residentes de dotación; el resto de los profesionales se hallaban ausentes debido a rotaciones, licencias y razones de servicio. Todos accedieron a responder el cuestionario. De las 480 encuestas obtenidas se descartaron 32 por errores en su confección, por lo que el análisis incluye los datos de 448 encuestas (Tabla 1).

La prevalencia de hábito de fumar entre los residentes de pediatría fue de $20,1 \%$ (IC 95\% 16,5 a 24,2$)(90 / 448)$. (Tabla 2).

La edad de comienzo de consumo de tabaco fue de 17,2 $\pm 2,9$ años. El 45,1\% de los encuestados identificaron los espacios abiertos como el lugar donde más fumaban dentro del hospital. Respecto del impacto de la incorporación al programa de residencia, 29,8\% refirieron fumar más después de incorporarse, mientras que $27,4 \%$ dejaron de hacerlo y $15,3 \%$ disminuyeron el consumo.

No se encontraron diferencias significativas entre fumadores y no fumadores en cuanto al sexo, tener hijos, el número de guardias semanales $\mathrm{y}$ tener un jefe directo fumador (Tabla 3).

$\mathrm{El}$ antecedente de tabaquismo en el padre fue un factor de riesgo para ser fumador solo en las mujeres (OR 1,98; IC 95\% 1,09 a 3,61; $p=0,01$ ).

Los que vivían en pareja fumaban menos que aquellos que vivían solos, con sus padres, parientes o amigos (15,4\% contra $23,4 \%$; $p=0,05$; OR 0,6; IC 95\% 0,25 a 1).

En cuanto a su actitud ante el tabaquismo de sus pacientes, $46,7 \%$ afirmaron que preguntaban siempre a los pacientes y a sus padres si fumaban; $37,7 \%$ advertían siempre acerca de los riesgos de consumir tabaco y $32,4 \%$ siempre aconsejaban abandonar el hábito o abstenerse de comenzar a fumar, presentando una conducta activa el $18,1 \%$ (preguntar, advertir y aconsejar siempre) (véase Tabla 2). Aquellos que habían recibido información durante su etapa de formación referían preguntar y aconsejar siempre con más frecuencia que aquellos que no lo habían hecho (OR 1,58; IC 95\% 1,05 a 2,38; $p=0,02$ y OR 1,63; IC $95 \% 1,04$ a 2,$55 ; p=0,02$, respectivamente), sin diferencias significativas en cuanto a advertir (OR 1,49; IC 95\% 0,97 a 2,28; $p=0,05$ ). No se observaron diferencias en la conducta al comparar los médicos fumadores con los no fumadores, pero aquellos que habían recibido información asumían con mayor frecuencia una conducta activa (OR 2,09; IC 95\% 1,20 a 3,65; $p=0,005$ ).

Análisis multivariado: al incorporar en un modelo multivariado edad, sexo, número de guardias, convivencia en pareja, padre fumador, madre fumadora y jefe fumador, solo la convivencia en pareja se mostró como un factor protector de ser fumador (OR 0,57; IC 95\% 0,34 a 0,$96 ; p=0,03$ ).

Se construyó otro modelo multivariado para evaluar la conducta activa, incorporando edad; sexo; tabaquismo en el padre, la madre y el

TABla 1. Distribución de los médicos residentes participantes según el hospital al que pertenecían

\begin{tabular}{lcccccc}
\hline Hospital & Ciudad & $\begin{array}{c}\text { Total de } \\
\text { residentes }\end{array}$ & Participantes & \multicolumn{2}{c}{ Porcentaje del total } \\
& & & $\mathbf{n}$ & $\mathbf{\%}$ & $\mathbf{2 0 1 1}$ & $\mathbf{2 0 0 2}$ \\
\hline Elizalde & Buenos Aires & 116 & 99 & 85,3 & 22,1 & 27,5 \\
Garrahan & Buenos Aires & 145 & 54 & 37,2 & 12,1 & 9,5 \\
Gutiérrez & Buenos Aires & 154 & 90 & 58,4 & 20,1 & 18,9 \\
Santísima Trinidad & Córdoba & 85 & 49 & 57,6 & 10,9 & 12,6 \\
Infantil & Córdoba & 53 & 51 & 96,2 & 11,4 & 10,0 \\
Sor María Ludovica & La Plata & 78 & 53 & 67,9 & 11,8 & 10,9 \\
Notti & Mendoza & 44 & 39 & 88,6 & 8,7 & 7,4 \\
Castro Rendón & Neuquén & 14 & 13 & 92,9 & 2,9 & 3,2 \\
Total & & 689 & 448 & 65,0 & 100,0 & 100,0 \\
\hline
\end{tabular}


médico residente; y haber recibido información; se encontró que solo recibir información constituía un predictor independiente de tener una conducta activa en cuanto al tabaquismo de los pacientes o

TABLA 2. Distribución de las variables evaluadas en la población estudiada $(n=448)$

\begin{tabular}{lc}
\hline Característica & Porcentaje \\
\hline Año de residencia & 25,7 \\
$1^{\text {er }}$ año & 25,7 \\
$2^{\text {o }}$ año & 28,6 \\
$3^{\text {er }}$ año & 83,3 \\
Sexo femenino & 20,1 \\
Fumadores & 25,5 \\
Vive solo & 40,7 \\
Vive en pareja & 8,5 \\
Tiene hijos & 45,5 \\
Madre fumadora & 60 \\
Padre fumador & 33,9 \\
Ambos padres fumadores & 71,7 \\
Padre o madre fumador & 47,3 \\
Jefe directo fumador & 63,6 \\
Recibió información & 46,7 \\
Pregunta siempre & 37,7 \\
Advierte siempre & 32,4 \\
Aconseja siempre & 18,1 \\
Conducta activa & \\
\hline
\end{tabular}

sus padres (OR 1,98; IC 95\% 1,16 a 3,36; $p=0,012$ ).

Comparación con el estudio anterior: no se observó una disminución significativa de la prevalencia del consumo de tabaco $(20,1 \%$ contra $22,1 \%$; OR $0,89$; IC 95\% 0,62 a 1,27; $p=0,5)$, pero sí mayor proporción de residentes que aconsejaban $(32,4 \%$ contra 26,1\%; OR 2,09; IC 95\% 1,48 a 2,96; $p=$ $0,00001)$ y advertían a sus pacientes $(37,7 \%$ contra $18,6 \%$; OR 1,76; IC 95\% 1,25 a 2,36; $p=0,0005)$. Se observó un incremento del porcentaje de mujeres en la residencia $(83,3 \%$ contra $76,2 \%$; OR 1,55 ; IC $95 \% 1,08$ a 2,$23 ; p=0,013$ ) y aumento del porcentaje de residentes que habían recibido información durante su formación (63,6\% contra 39,8\%; OR 2,67; IC 95\% 1,98 a 3,60; $p<0,001$ ) (Tabla 4).

\section{DISCUSIÓN}

Encontramos que 20,1\% de los residentes de pediatría eran fumadores. A pesar de las medidas implementadas para disminuir la exposición al tabaco y su consumo en los últimos años, la prevalencia del tabaquismo en la población estudiada no se modificó sustancialmente en relación con lo evaluado en $2002(22,1 \%) .^{7}$ Esta tendencia es similar a la observada a nivel nacional en el mismo grupo etario ( 25 a 34 años) entre $2005(34,6 \%)^{11}$ y $2009(33,3 \%){ }^{10}$

Si se compara con lo referido por Haddock ${ }^{12}$ en residentes de pediatría de Nueva Jersey $(7 \%)$, la prevalencia del tabaquismo en los residentes

TABLA 3. Comparación de la distribución de potenciales predictores entre médicos residentes fumadores actuales y no fumadores

\begin{tabular}{|c|c|c|c|c|c|}
\hline Características & Fumadores & No fumadores & OR & IC $95 \%$ & $p$ \\
\hline Edad (años) & $28,76 \pm 2,05$ & $28,37 \pm 2,08$ & & & 0,11 \\
\hline Sexo femenino & $84,4 \%$ & $83 \%$ & 1,11 & $0,57-2,21$ & 0,74 \\
\hline Guardias (2 o más) & $81,1 \%$ & $72 \%$ & 1,66 & $0,91-3,09$ & 0,08 \\
\hline Vive solo & $32,2 \%$ & $23,8 \%$ & 1,52 & $0,89-2,59$ & 0,1 \\
\hline Vive en pareja & $31,1 \%$ & $43,1 \%$ & 0,60 & $0,35-1,00$ & 0,03 \\
\hline Tiene hijos & $7,8 \%$ & $8,7 \%$ & 0,89 & $0,34-2,21$ & 0,79 \\
\hline Madre fumadora & $50 \%$ & $44,4 \%$ & 1,25 & $0,77-2,04$ & 0,34 \\
\hline Padre fumador & $68,9 \%$ & $57,8 \%$ & 1,62 & $0,96-2,73$ & 0,05 \\
\hline Ambos padres fumadores & $38,9 \%$ & $32,7 \%$ & 1,31 & $0,79-2,17$ & 0,27 \\
\hline Madre o padre fumador & $80 \%$ & $69,5 \%$ & 1,75 & $0,97-3,20$ & 0,04 \\
\hline Mujer - padre fumador & $73,7 \%$ & $58,6 \%$ & 1,98 & $1,09-3,61$ & 0,01 \\
\hline Mujer - madre fumadora & $52,6 \%$ & $44,8 \%$ & 1,37 & $0,80-2,34$ & 0,22 \\
\hline Hombre - padre fumador & $42,8 \%$ & $54,1 \%$ & 0,64 & $0,17-2,35$ & 0,45 \\
\hline Hombre - madre fumadora & $35,7 \%$ & $42,6 \%$ & 0,75 & $0,19-2,85$ & 0,64 \\
\hline Jefe directo fumador & $48,9 \%$ & $46,9 \%$ & 1,08 & $0,66-1,76$ & 0,74 \\
\hline Recibió información & $61,1 \%$ & $64,2 \%$ & 0,87 & $0,53-1,45$ & 0,58 \\
\hline Conducta activa & $21,1 \%$ & $20,4 \%$ & 1,04 & $0,57-1,91$ & 0,88 \\
\hline
\end{tabular}


de pediatría en la Argentina es elevada. Sin embargo, está por debajo de la comunicada tanto para estudiantes de medicina como para médicos argentinos. El estudio FUMAr, ${ }^{13}$ realizado en el año 2004 en 12 facultades de medicina de la Argentina, estimó una prevalencia de tabaquismo en los estudiantes de $35,2 \%$, mientras que el estudio TAMARA ${ }^{14}$ informó una prevalencia del 30,2\% en una muestra de casi 6500 médicos argentinos.

Pese a los conocimientos sobre las consecuencias del consumo de tabaco y el contacto permanente con la morbimortalidad que genera, la diferencia de porcentajes de fumadores entre los médicos y la población general continúa siendo baja en la Argentina. En los países desarrollados esta diferencia es mucho mayor, con prevalencias de tabaquismo en los médicos y en la población general de $6 \%$ y $40 \%$ respectivamente, en Holanda, ${ }^{15,16} 5,5 \%$ y $23 \%$ en Finlandia, ${ }^{17,18} 17 \%$ y $35 \%$ en Japón, ${ }^{18,19}$ y $4 \%$ y $23 \%$ en los Estados Unidos. . $^{18,20}$

A diferencia del estudio de 2002, en el que se observó un incremento del consumo de tabaco después del ingreso en la residencia (38,9\% fumaban más después de ingresar), en el presente estudio este porcentaje descendió a 29,8\%. Esto podría deberse, en parte, a la aplicación de algunas medidas en los últimos años destinadas a mejorar la calidad de vida del residente, como la disminución del número de guardias realizadas o la implementación del descanso luego de una guardia de 24 horas en varios hospitales. ${ }^{21}$ Pese a que los estudios realizados en la Argentina analizan el consumo de tabaco en médicos de distintas especialidades, ${ }^{14}$ no existe información en nuestro medio sobre el tabaquismo en residentes de otras especialidades que permita realizar un análisis comparativo con la población estudiada.

Casi la mitad de los fumadores $(45,1 \%)$ identificaron los espacios abiertos como el lugar donde más fumaban dentro del hospital. Aunque en algunos de los distritos incluidos no se prohíbe el consumo de tabaco en lugares abiertos, en otros, como la Ciudad Autónoma de Buenos Aires (a la que pertenece más del $50 \%$ de la población estudiada), la prohibición en los establecimientos de salud es absoluta. ${ }^{22}$

Se han implicado factores genéticos, ambientales y culturales en el consumo de tabaco. En el presente estudio se encontró que tener un padre fumador constituía un factor de riesgo, en consonancia con la evidencia que muestra que el tabaquismo de los padres constituye un factor de riesgo para el inicio del consumo en los adolescentes. ${ }^{23-26}$

Resulta difícil comparar la actitud preventiva de los residentes (preguntar, advertir, aconsejar) con otras experiencias, ya que esta información fue recabada de manera diferente en distintos estudios. No obstante, al igual que lo observado por otros autores. ${ }^{12,27}$ encontramos que los residentes aconsejaban y advertían menos de lo que preguntaban, conductas que requieren mayor conocimiento y habilidades sobre el cese del tabaquismo. En coincidencia con ello, se observó que aquellos que habían recibido información durante la carrera de medicina o la residencia eran más proclives a tomar una conducta activa sobre la cesación del tabaquismo de sus pacientes, preguntando, aconsejando y advirtiendo más que aquellos que no lo habían hecho. Barnes Dodge $^{27}$ halló que $83 \%$ referían preguntar, pero solo $36 \%$ aconsejaban dejar de fumar a los pacientes o cuidadores, y que el conocimiento de los recursos para dejar de fumar era el factor que más se asociaba con esa conducta. Existe evidencia de que el entrenamiento específico en la materia incrementa la conducta activa de los profesionales frente al tabaquismo de los pacientes. ${ }^{28-30}$ Debe tenerse en cuenta que, por tratarse de una encuesta realizada a los médicos, podría estar sobreestimada la frecuencia

TABLA 4. Comparación con los resultados de la encuesta realizada en $2002^{7}$

\begin{tabular}{lccccc}
\hline & $\mathbf{2 0 0 2}$ & $\mathbf{2 0 1 1}$ & OR & IC $\mathbf{9 5} \%$ & $p$ \\
\hline Fumar & $22,1 \%$ & $20,1 \%$ & 0,89 & $0,62-1,27$ & 0,5 \\
Sexo femenino & $76,2 \%$ & $83,3 \%$ & 1,55 & $1,08-2,23$ & 0,013 \\
Recibió información & $39,8 \%$ & $63,6 \%$ & 2,67 & $1,98-3,60$ & $<0,001$ \\
Pregunta siempre & $39,5 \%$ & $46,4 \%$ & 1,33 & $0,99-1,78$ & 0,05 \\
Advierte siempre & $18,6 \%$ & $37,7 \%$ & 1,72 & $1,25-2,36$ & $<0,001$ \\
Aconseja siempre & $26,1 \%$ & $32,4 \%$ & 2,09 & $1,48-2,96$ & $<0,001$ \\
\hline
\end{tabular}


con que estos presentan conductas activas. Pocos estudios recaban estos datos a través de los pacientes: en dos estudios realizados por Hymowitz, ${ }^{28,31}$ se informa que solo un $10 \%$ y un $21 \%$ de los fumadores respectivamente referían que su médico les ofreció ayuda para dejar de fumar. El incremento observado en las conductas analizadas (preguntar, advertir y aconsejar) podría estar relacionado con una mayor proporción de profesionales que habían recibido información sobre el cese del tabaquismo.

La elevada proporción de sujetos que refieren haber recibido información sobre el tabaquismo (63\%) contrasta con el 5,2\% de los alumnos de medicina que refieren haber recibido entrenamiento formal en abordajes para la cesación del hábito durante sus estudios en la encuesta global sobre tabaquismo en estudiantes de las profesiones de la salud (GHPSS, por su sigla en inglés), lo que evidencia un déficit en la formación de pregrado. ${ }^{32}$ Esta diferencia puede explicarse porque nosotros preguntamos sobre haber recibido información y no sobre haber sido entrenado formalmente, destacando la diferencia entre adquirir conocimientos y competencias. Además, pese a no haber sido un dato recabado en la encuesta, en la mayoría de los centros participantes no existen herramientas concretas para una intervención efectiva sobre el cese del tabaquismo, hecho que limita la posibilidad de intervención real sobre la exposición al humo de tabaco.

Es posible que los escasos cambios en el consumo de tabaco en los médicos residentes de pediatría en la Argentina estén relacionados con que, si bien el país adhirió al CMCT en 2003, aún no ha ratificado su adhesión al convenio ni ha cumplido completamente con sus postulados.

\section{CONCLUSIONES}

El 20,1\% de los médicos residentes de pediatría eran fumadores, siendo más frecuente en las mujeres con un padre fumador. La conducta activa frente al tabaquismo de los pacientes o sus padres fue muy baja. Pese a las políticas oficiales, el tabaquismo en este grupo no se ha modificado en la última década, aunque aumentó la proporción de quienes recibieron información durante su formación, y aconsejan y advierten a los pacientes sobre sus riesgos.

\section{BIBLIOGRAFÍA}

1. World Health Organization. WHO global report mortality attributable to tobacco. Geneva: World Health Organization; 2012. [Consulta: 1 de junio de
2012]. Disponible en: http://whqlibdoc.who.int/ publications/2012/9789241564434_eng.pdf.

2. Argentina. Ministerio de Salud y Ambiente de la Nación. Costos Directos de la Atención médica de las enfermedades atribuibles al consumo de tabaco en Argentina. Proyecto VIGIA. Buenos Aires: Ministerio de Salud y Ambiente de la Nación; 2005. [Consulta: 1 de junio de 2012]. Disponible en: http://msal.gov.ar/htm/site_tabaco11/pdf/costos_ directos_at_medica.pdf.

3. Patiño C. El rol del médico en la lucha antitabáquica. Alerg Inmunol Clin 2000;17(Suppl 1):s26.

4. Stein RJ, Haddock CK, O'Byrne KK, Hymowitz N, Schwab $\mathrm{J}$. The pediatrician's role in reducing tobacco exposure in children. Pediatrics 2000;106(5):e66.

5. Grupo Tabaquismo. Consenso sobre factores de riesgo de enfermedad cardiovascular en pediatría. Tabaquismo. Arch Argent Pediatr 2005;103(5):464-75.

6. Moreno MA. Resident stress revisited: a senior pediatric resident's point of view. Pediatrics 2003;112(2):411-4.

7. Ferrero F, Castaños C, Durán P, Blengini MT. Prevalencia del consumo de tabaco en médicos residentes de pediatría en Argentina. Rev Panam Salud Pública 2004;15(6):395-9.

8. Organización Mundial de la Salud. Convenio Marco de la OMS para el Control del Tabaco. WHO Document Production Services; 2003. [Consulta: 1 de junio de 2012]. Disponible en: http://whqlibdoc.who.int/ publications/2003/9243591010.pdf.

9. Secretaría de Programación para la prevención de la Drogadicción y la Lucha contra el Narcotráfico (SEDRONAR). Estudio Nacional sobre consumo de sustancias adictivas. Buenos Aires, Argentina; 1999.

10. Ferrante D, Linetzky B, Konfino J, King A, et al. Encuesta Nacional de Factores de Riesgo 2009: Evolución de la epidemia de enfermedades crónicas no transmisibles en Argentina. Estudio de corte transversal. Rev Argent Salud Pública 2011;2(6):34-41.

11. Argentina. Ministerio de Salud de la Nación. Encuesta Nacional deFactores de Riesgo 2005-Informede Resultados. Argentina; 2005. [Consulta: 1 de junio de 2012]. Disponible en: http://msal.gov.ar/ENT/VIG/Areas_Tematicas/ Factores_de_Riesgo/PDF/Encuesta $\% 20$ Nacional $\% 20$ de $\% 20$ Factores\%20de\%20Riesgo\%202005_informe_final_ breve.pdf.

12. Haddock CK, Pyle S, Hymowitz N, Schwab J, et al. Which pediatric residents assist and arrange follow-up for patients and parents who use tobacco? J Adolesc Health 2005;36(6):531-3.

13. Zabert G, Verra F, Kevorkof G, Lungo B, et al. Estudio F.U.M.Ar (Fumar en Universitarios de Medicina en Argentina). Informe final. 2004. [Consulta: 1 de junio de 2012]. Disponible en: http://www.msal.gov.ar/tabaco/ images/stories/info-equipos-de-salud/pdf/paper-fumar2004v4-CAEM-2005.pdf.

14. Zylbersztejn HM, Cardone A, Vainstein N, Mulassi A, et al. Tabaquismo en médicos de la República Argentina: Estudio TAMARA. Rev Argent Cardiol 2007;75(2):109-16.

15. Kotz D, Wagena EJ, Wesseling G. Smoking cessation practices of Dutch general practitioners, cardiologists, and lung physicians. Respir Med 2007;101(3):568-73.

16. World Health Organization. Highlights on Health in the Netherlands. World Health Organization; 1997. [Consulta: 1 dejunio de 2012]. Disponible en: http:/ / www.euro.who. int/_data/assets/pdf_file/0018/130536/E62040.pdf.

17. Barengo NC,Sandström PH, Jormanainen VJ,Myllykangas MT. Changes in smoking prevalence among Finnish physicians 1990-2001. Eur J Public Health 2004;14(2):201-3.

18. Shafey O, Dolwick S, Guindon E. Tobacco Control Country Profiles 2003. Atlanta, GA: American Cancer Society; 2003. 
19. Ohida T,Sakurai H,Mochizuki Y,Kamal AM, etal.Smoking prevalence and attitudes toward smoking among Japanese physicians. JAMA 2001;285(20):2643-8.

20. Lee DJ, LeBlanc W, Fleming LE, Gómez-Marín O, et al. Trends in US smoking rates in occupational groups: the National Health Interview Survey 1987-1994. J Occup Environ Med 2004;46(6):538-48.

21. Ley $\mathrm{N}^{\mathrm{o}}$ 601. Modificación del Régimen de Residencias. Boletín Oficial de la Ciudad de Buenos Aires No 1232. Buenos Aires, Argentina, 13 de julio de 2001.

22. Ley $\mathrm{N}^{\circ} 1799$. Ley de Control del Tabaco. Boletín Oficial dela Ciudad de Buenos Aires N ${ }^{\circ}$ 2313. Buenos Aires, Argentina, 8 de noviembre de 2005.

23. Cogollo-Milanés Z, La Hoz-Restrepo F de. Consumo de cigarrillo y riesgo de dependencia de la nicotina de estudiantes de secundaria. Rev Salud Publica (Bogotá) 2010;12(3):434-45.

24. Giannakopoulos G,Tzavara C,DimitrakakiC,Kolaitis G, et al. Emotional, behavioural problems and cigarette smoking in adolescence: findings of a Greek cross-sectional study. BMC Public Health 2010;10:57.

25. Bricker JB, Peterson AV, Robyn Andersen M, Leroux BG, et al. Close friends', parents', and older siblings' smoking: reevaluating their influence on children's smoking. Nicotine Tob Res 2006;8(2):217-26.

26. FergussonDM,HorwoodLJ, BodenJM,Jenkin G.Childhood social disadvantage and smoking in adulthood: results of a 25-year longitudinal study. Addiction 2007;102(3):475-82.

27. Barnes Dodge RA, Cabana MD, O'Riordan MA, Heneghan A. What factors are important for pediatric residents' smoking cessation counseling of parents? Clin Pediatr (Phila) 2008;47(3):237-43.

28. Hymowitz N, Schwab JV, Haddock CK, Pyle SA, et al. The pediatric residency training on tobacco project: four-year resident outcome findings. Prev Med 2007;45(6):481-90.

29. Collins RL, D'AngeloS, Stearns SD, Campbell LR. Training pediatric residents to provide smoking cessation counseling to parents. Scientific World Journal 2005;5:410-9.

30. Lee MT, Hishinuma ES, Derauf C, Guerrero APS, et al. Smoking cessation counseling training for pediatric residents in the continuity clinic setting. Ambul Pediatr 2004;4(4):289-94.

31. Hymowitz N, Schwab J, Haddock CK, Pyle S, et al. The pediatric resident training on tobacco project: baseline findings from the Parent/Guardian Tobacco Survey. Prev Med 2005;41(1):334-41.

32. Global Health Professions Student Survey (GHPSS). FACT SHEET Argentina - Medical Students (3rd Year Students Only). [Consulta: 1 de junio de 2012]. Disponible en: http://new.paho.org/hq/dmdocuments/2010/2005\%20 Argentina $\% 20$ GHPSS $\% 20 \% 28$ Medical $\% 29 \% 20$ Factsheet. pdf. 